DOI: $10.23857 / p c . v 2 i 3.297$

Recepción: 21 / 09 / 2016

Aceptación: 16 / 01 / 2017

Publicación: 06 / 03 / 2017

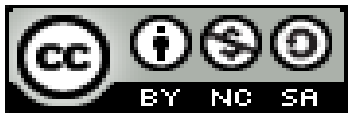

Ciencias técnicas y aplicadas

Artículo original

\title{
Implementación de un sistema automatizado de bombeo de agua para la generación de oxígeno artificial utilizando energía solar para piscicultura de la finca "El Porvenir"
}

\begin{abstract}
Implementation of an automated water pumping system for the generation of artificial oxygen using solar energy for pisciculture of the estate "El Porvenir"
\end{abstract}

\section{Implementação de um sistema automático de bombeamento da água para a geração de oxigénio artificial utilizando a energia solar para a aquicultura fazenda "El Porvenir"}

\author{
Alex F. Lema-Ilguán ${ }^{\mathrm{I}}$ \\ a_lema07@hotmail.com \\ Sergio F. Padilla-Calle II \\ spg_kd34@hotmail.com \\ Edwin V. Altamirano-Santillán III \\ ealtamirano@espoch.edu.ec \\ Geovanny E. Vallejo-Vallejo ${ }^{\text {IV }}$ \\ g_vallejo@espoch.edu.ec \\ Hugo M. Velastegui-Noboa ${ }^{\mathrm{V}}$ \\ hugo.velastegui@espoch.edu.ec
}

Correspondencia:1veloz@espoch.edu.ec

\footnotetext{
${ }^{\mathrm{I}}$ Ingeniero en Electrónica, Control y Redes Industriales, Escuela Politécnica de Chimborazo, Riobamba, Ecuador.

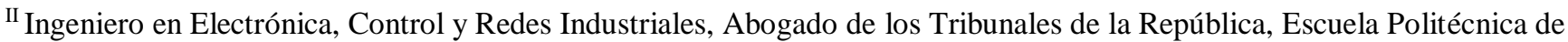
Chimborazo, Riobamba, Ecuador.

III Magister en Gestión Académica Universitaria, Diplomado Superior en Docencia Universitaria, Ingeniero en Electrónica y Computación, Docente en Escuela Politécnica de Chimborazo, Riobamba, Ecuador.

IV Magister en Docencia Universitaria e Investigación Educativa, Doctor en Ciencias de la Educación Mención Informática Educativa, Licenciado en Ciencias de la Educación Mención Informática Educativa, Docente de Escuela Superior Politécnica de Chimborazo, Riobamba, Ecuador.

${ }^{\mathrm{v}}$ Magister en Pequeñas y Medianas Empresas Mención Finanzas, Master Internacional en Turismo, Licenciado en Ciencias Administrativas, Ingeniero Comercial. Docente de Escuela Superior Politécnica de Chimborazo, Riobamba, Ecuador.
} 


\section{Resumen}

El objetivo fue implementar un sistema automatizado de bombeo de agua para generación de oxígeno artificial utilizando energía solar para piscicultura de la finca "El Porvenir". La investigación hace énfasis sobre el uso de la energía solar fotovoltaica por el beneficio que presenta con respecto al impacto ambiental en la reducción del $\mathrm{CO} 2$, mediante la utilización de fuentes de energías renovables. Con el uso del Atlas Solar del Ecuador se realizó el análisis de radiación del sitio donde se implementó el proyecto, luego se procedió al dimensionamiento del sistema en cada uno de las etapas de implementación. Dentro de estos procesos se realizó: la orientación e inclinación del módulo fotovoltaico, dimensionamiento del inversor DC - AC, dimensionamiento de la bomba y selección de los equipos a utilizar. Con la información proporcionada por el sensor de oxígeno el Arduino PLC, permitió el control del sistema aumentando o disminuyendo la potencia del motor. El sensor de oxígeno permitió monitorear la cantidad de oxígeno disuelto en el estanque. Los resultados obtenidos de la medición de oxígeno, temperatura, caudal y presión permitieron determinar la concentración de oxígeno necesario en el estanque en el valor de $6.525 \mathrm{ppm}$, garantizando la producción de oxígeno apta para la crianza de peces, logrando mejores beneficios para el sector piscicultor. Con el uso de la energía fotovoltaica como una opción para generar fuente de energía se logró eliminar el $\mathrm{CO} 2$ en un $100 \%$ de forma que se contribuye a la no contaminación e impacto ambiental. Al momento de seleccionar los paneles solares se recomienda lo de tipo mono cristalinos por la mayor tasa de eficiencia y condiciones de rendimiento con respecto a las demás y su vida útil es de 25 años.

Palabras clave: radiación solar; dimensionamiento de la instalación; módulo fotovoltaico; sensor de oxígeno; oxígeno disuelto; arduino PLC.

\section{Abstract}

The objective was to implement an automated water pumping system for artificial oxygen generation using solar energy for fish-farming of the Porvenir farm. The research emphasizes the use of photovoltaic solar energy for the benefit it presents with respect to the environmental impact in the reduction of $\mathrm{CO} 2$ through the use of renewable energy sources. The radiation analysis of the place where the project was implemented has been made with the use of the Solar Atlas of Ecuador, the proceeded to sizing of the system in each one of the stages of implementation. Within these processes was carried out: the orientation and inclination of the 
photovoltaic module, inverter sizing of the DC-AC, sizing of the pump and selection of the equipment to be used. With the information provided by the oxygen sensor Arduino PLC that allowed to monitor the amount of temperature, flow and pressure allowed to determine the concentration of oxygen required in the pond in the value of $6,525 \mathrm{ppm}$, guaranteeing the production of oxygen suitable for fish farming, achieving better benefits for the fish breeder sector. It was possible to eliminate the CO2 in the $100 \%$ with the uses of photovoltaic energy as an option to generate energy source so that contributes to non-pollution and environmental impact. At the time of selecting the solar panels, the mono-crystalline type is recommended for the highest efficiency rate and performance conditions compared to the others and its useful life is 25 years.

Keywords: Solar radiation; sizing of the place; photovoltaic module; oxygen sensor; dissolved oxygen; arduino PLC.

\section{Resumo}

O objetivo foi implementar um sistema automatizado para bombear água para a geração de oxigénio artificial utilizando a energia solar para a criação de peixes fazenda "El Porvenir". A pesquisa enfatiza o uso da energia solar fotovoltaica para o benefício apresentado em relação ao impacto ambiental sobre a diminuição do $\mathrm{CO} 2$ usando fontes de energia renováveis. Com o uso do Atlas do Equador análise radiação solar do local onde o projeto foi implementado foi conduzido, em seguida, ele seguiu para o dimensionamento do sistema em cada etapa da implementação. Dentro destes processos foi realizada: a orientação e inclinação do módulo fotovoltaico, dimensionando inversor DC - AC, dimensionamento da bomba e seleção de equipamentos de usar. Com as informações fornecidas pelo sensor de oxigênio PLC Arduino, que permitiu o controle de aumentar ou diminuir o sistema de alimentação do motor. O sensor de oxigénio permitido controlar a quantidade de oxigénio dissolvido na lagoa. Os resultados da medição de oxigénio, temperatura, fluxo e pressão permitida para determinar a concentração de oxigénio requerida na lagoa no valor de $6.525 \mathrm{ppm}$, garantindo a produção de oxigénio apropriado para a criação de peixes, a obtenção de melhores benefícios para a indústria de agricultor . Com o uso da energia fotovoltaica como fonte de energia alternativa para gerar conseguiu eliminar CO2 em $100 \%$ para que ele contribui para a não-poluição e impacto ambiental. Ao selecionar painéis solares recomendou o cristalino mono para a maior taxa de 
condições de eficiência e desempenho com respeito aos outros ea expectativa de vida é de 25 anos.

Palavras chave: radiação solar; dimensionamento da instalação; módulo fotovoltaico; sensor de oxigênio; oxigénio dissolvido; arduino PLC.

\section{Introducción}

Una de las formas más comunes de cultivar peces es mediante criaderos en piscícolas o estanques, por lo cual en estas piscícolas es muy importante disponer de mecanismos de producción de oxígeno, el más común y usado en la actualidad es provocando una caída de agua a la piscina.

Dicho de agua se asemeja a la producción de oxígeno que encontramos en los ríos de forma natural. Para el proceso de crianza de peces uno de los principales factores y muy importantes en su estado de crecimiento es una gran cantidad de oxígeno disuelto, en épocas de invierno por la gran cantidad de caudal en las vertientes, la cantidad de oxígeno disuelto en las piscícolas es estable.

El problema se genera cuando se presenta una disminución de caudal en la temporada de verano, provocando así la muerte de los peces debido a la reducción del nivel de oxígeno disuelto; ocasionando pérdidas económicas a los piscicultores. Una forma de contraer el problema es bombear agua con motores de combustión generando gastos y contaminación ambiental al utilizar combustibles fósiles. Un factor importante a tomar en cuenta también es detectar a tiempo la pérdida de oxígeno para evitar la muerte de los peces. Con el presente trabajo de investigación se pretende automatizar el proceso mediante el aprovechamiento de la energía solar fotovoltaica.

Hoy en la actualidad las fuentes de energías renovables se han convertido en los pilares primordiales de una política energética, debido a la sustentabilidad que posee, por ello el presente trabajo de investigación tiene como objetivo desarrollar proyectos tecnológicos a base de la energía fotovoltaica, mediante la implementación de un sistema de bombeo de agua para la generación de oxígeno artificial utilizando energía solar en beneficio de la piscicultura.

De esta manera ser promotores capaces de preservar las fuentes de energía, y sin crear un ningún impacto ambiental, así también ayudando a la protección, seguridad del medio ambiente. 


\section{Fases del diseño}

Para el diseño e implementación del proyecto, se toman en cuenta los siguientes aspectos: ubicación, diseño estructural del soporte de los paneles fotovoltaicos, diseño y dimensionamiento del sistema de generación FV y eléctrico, implementación del variador de frecuencia, programación del Arduino PLC, implementación del HMI y de los dispositivos de control y mando.

\section{Ubicación del proyecto}

El lugar donde se va instalar el sistema de generación fotovoltaica es en la Provincia de Cañar, en la Parroquia de Zhud, de coordenadas geográficas son: latitud: -2.460696, longitud: -79.004574.

\section{Dimensionamiento de la bomba}

En la figura 1 se muestra el diseño del sistema de bombeo teniendo en cuenta el caudal diario de agua requerida, la altura total y el lugar de la instalación. El caudal requerido es de $18 \mathrm{~m} 3 / \mathrm{h}$ en base al tanque de purificación además se tomó en cuenta el volumen del estanque de la crianza de peces que es de $60 \mathrm{~m} 3$, altura de succión desde de $1.5 \mathrm{~m}$ por debajo de la cota de terreno, hasta una altura de $2 \mathrm{~m}$.

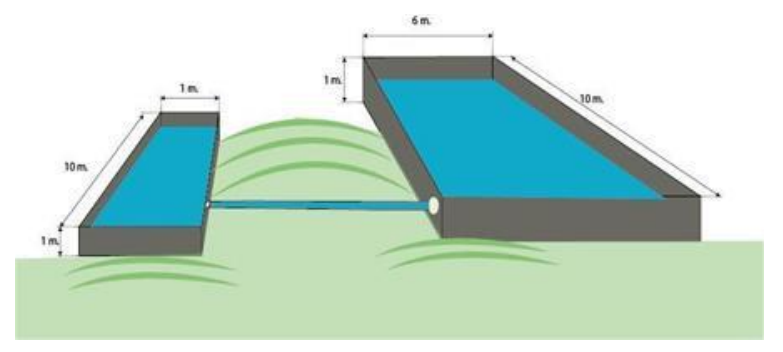

Figura 1. Dimensiones del estanque

El diámetro de tubería utilizado tanto para la línea de aspiración y succión de caudal es de 50 $\mathrm{mm}$, determinado los datos. Para el proyecto se desea seleccionar una bomba centrifuga, por sus múltiples ventajas que ofrece para este tipo de aplicaciones, por ser mecanismos diseñados para bombear agua desde un depósito a una altura determinada. Con los parámetros y características del sistema de bombeo se tiene el siguiente diseño como se muestra en la figura 2. 


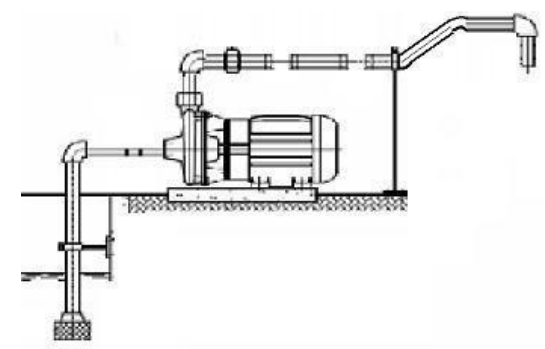

Figura 2. Diseño del sistema de bombeo

\section{Cálculo de la altura manométrica}

Se realizó mediante la ecuación 1 tal como se muestra a continuación.

Ecuación 1. Altura manométrica

$$
H=H_{g}+P_{c}+10 \frac{P i-P a}{\gamma}
$$

Donde:

$\mathrm{H}$ : Altura manométrica

Hg: Altura geométrica

Pc: La pérdida de carga del flujo por paso de la tubería.

$(P i-P a) / \gamma:$ La presión diferencial entre la superficie de salida y de entrada de la bomba.

\section{Determinación de las pérdidas de carga}

Para determinar la pérdida de carga tanto en la entrada y salida de la tubería primero se debe calcular la longitud real Leq más los accesorios tales como: codos, válvulas de pie/retención.

Una vez encontrada la Leq se obtiene la pérdida de la carga, consiste la sección algunos procedimientos más simplificados y rápidos, permitiendo estimar las pérdidas de carga que se originan en una instalación de bombeo de agua. Para lo cual se usa la tabla donde están tabuladas las pérdidas de carga por rozamiento, expresadas en metros para tramos rectos de tuberías de 100 metros de longitud, en función del caudal que circula y su diámetro interior:

Tubería de entrada: para encontrar la Leq de la tubería de entrar se utilizó la ecuación 2:

$$
\text { Leq }=\text { longitud tubería }+ \text { longitud equivalente válvula de pie }
$$




$$
L e q=1.5 m+9 m=10.5 m
$$

Tubería de salida: para encontrar la Leq de la tubería de salida se utilizó la ecuación 3:

$$
\begin{gathered}
\text { Leq }=\text { longitud tubería }+ \text { longitud codo } 900+\text { longitud codo } 450 \\
\qquad \text { Leq }=3.5 \mathrm{~m}+(2 * 1.5) \mathrm{m}+(2 * 1) \mathrm{m}=8.5 \mathrm{~m}
\end{gathered}
$$

La pérdida de carga para $18 \mathrm{~m}^{3} / \mathrm{h}$ en tubería de $50[\mathrm{~mm}]$ en la entrada y la salida es de:

$$
\begin{aligned}
& P_{\text {c in }}=\frac{10.95 * 10.5}{100}=1.15 \mathrm{~m} \\
& P_{\text {c out }}=\frac{10.94 * 8.5}{100}=0.93 \mathrm{~m}
\end{aligned}
$$

Altura manométrica de entrada se calculó con la ecuación 1 tomado en cuenta que $(P i-P a) / \gamma$ es cero por tratarse de depósitos abiertos a la atmósfera.

$$
\begin{gathered}
\text { H entrada }=\mathrm{H}_{\mathrm{a}}+\mathrm{P}_{\mathrm{ca}} \\
\mathrm{H} \text { entrada }=1+1.15=2.15 \mathrm{~m} \\
\mathrm{H} \text { salida }=\mathrm{H}_{\mathrm{a}}+\mathrm{P}_{\mathrm{ci}} \\
\mathrm{H} \text { salida }=2+0.93=2.93 \mathrm{~m} \\
\mathrm{H} \text { total }=\mathrm{H} \text { entrada }+\mathrm{H} \text { salida } \\
\mathrm{H} \text { total }=2.15+2.93=\mathbf{5 . 0 8} \mathbf{~ m}
\end{gathered}
$$

\section{Selección de la bomba}

Para la selección de la bomba, cada fabricante dispone de una tabla de selección rápida que permite obtener el modelo de bomba y las prestaciones que ofrece de entre toda la gama de bombas que se presentan. En estas tablas se encuentran los valores de caudal y altura manométrica que debe suministrar la bomba, y que han sido calculados previamente. En este caso, se ha optado por seleccionar el equipo de bombeo del fabricante LEO, de cuyos catálogos se puede extraer la siguiente tabla de selección rápida de bombas.

Como se muestra en la figura 3, entrando en la tabla con un caudal $(\mathrm{Q}=18 \mathrm{~m} 3 / \mathrm{h})$ y una altura manométrica $(\mathrm{H}=5.08 \mathrm{~m})$ resulta adecuado el modelo de la bomba XKP804A de $1 \mathrm{HP}$ potencia, de la gama de bombas de fabricante de la marca LEO. 


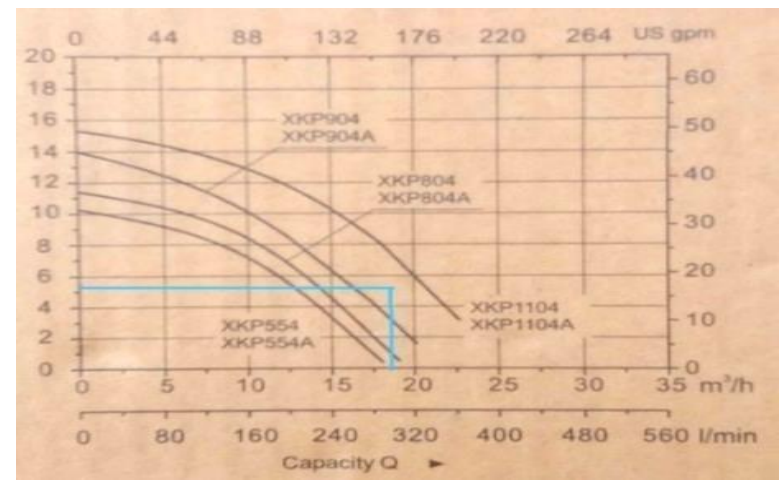

Figura 3. Tabla de selección de bombas

La bomba seleccionada para este proyecto es como se muestra en la figura 4.

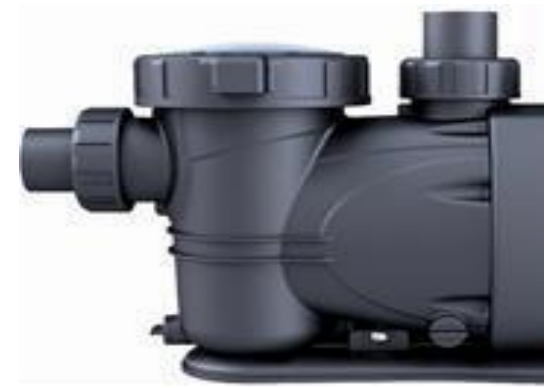

Figura 4. Bomba Centrifuga marca LEO

\section{Montaje y alineación de la bomba con el eje del motor}

En la figura 5 se puede ver el acoplamiento realizado entre el eje de la bomba y el eje del motor a través del acople de mandíbula, de forma que todo el sistema quede empotrado de forma alineada para una mejor funcionalidad del sistema. La plataforma de empotre del motor y la bomba está diseñado con tubos de acero inoxidable.

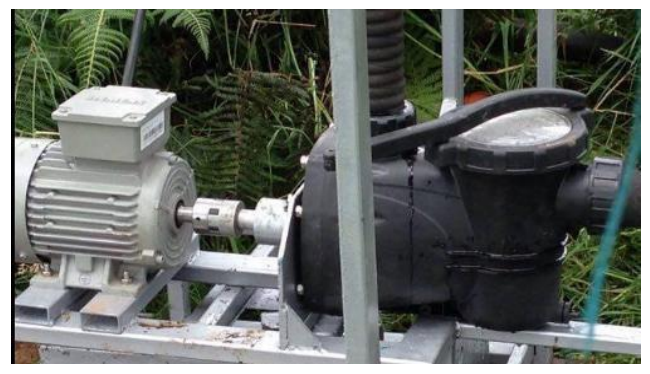

Figura 5. Conjunto bomba motor 
El sistema de acople entre el eje de la bomba cuenta con un sistema de lubricación para inyectar grasa al rodamiento instalado, como se muestra en la figura 6, con la finalidad de reducir el desgaste garantizando de esta manera aumentar la vida útil del rodamiento.

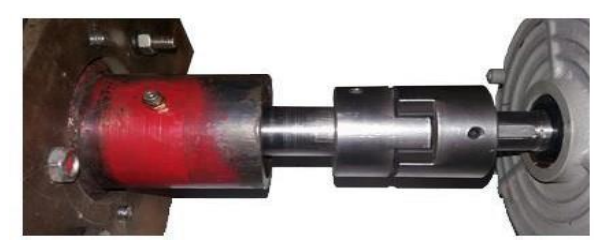

Figura 6. Sistema de mantenimiento

\section{Diseño y dimensionamiento del sistema de generación fotovoltaico}

Para realizar el dimensionamiento se determinó la radiación solar donde se va instalar el proyecto, para ello se tomó en cuenta los datos proporcionados por el Atlas Solar del Ecuador del CONELEC con fines de generación eléctrica. En la zona de la provincia de Cañar se registra una insolación directa promedio anual de $2800 \mathrm{Wh} / \mathrm{m} 2 /$ día.

Selección de equipos

Una vez determinado la radiación del sitio, la potencia requerida por el motor de $1 \mathrm{HP}$ y tomando tomar en cuenta las horas diarias de trabajo. En este caso se ha optado por seleccionar paneles solares de tecnología mono-cristalino Techno Suzhou, modelo SM636-150 por su alta eficiencia de célula del $17.96 \%$, cuenta con una tolerancia del $\sim+3 \%$ en relación al poli-cristalino u otras tecnologías similares. La figura 7 muestra el panel solar mono- cristalino.

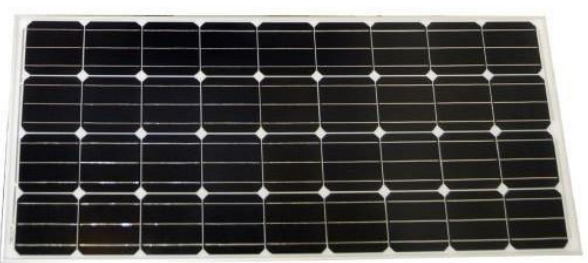

Figura 7. Panel mono-cristalino

A continuación, se determina el cálculo matemático para el dimensionamiento del generador FV en base a las especificaciones antes mencionadas.

Demanda de energía del motor de la bomba: se calculó mediante la ecuación 2, tomando en cuenta los siguientes datos: 
- $\mathrm{P}=1 \mathrm{HP}=746 \mathrm{~W}$

$\cdot \mathrm{t}=8$ horas

Ecuación 2: Demanda de energía del motor de la bomba

$\mathrm{E}=\mathrm{P} * \mathrm{t}=746 \mathrm{~W} * 8 \mathrm{~h}=\mathbf{6 0 0 0} \mathrm{Wh} /$ día

\section{Cálculo del número de paneles a utilizarse en el proyecto}

Este cálculo se realizó mediante una vez hallado el factor de paneles con la ecuación 3, tomando en cuenta la energía requerida y el factor del panel, una vez obtenido este factor se procedió hallar el número de paneles requerido mediante la ecuación 4.

- Energía requerida: 6000Wh/día

- Potencia del panel: $150 \mathrm{~W}$

Ecuación 3: Factor de paneles

$$
\begin{gathered}
\text { Factor de paneles } \\
=\frac{E}{\text { P paneles }}+\frac{E}{\text { P paneles }} * 0.25 \\
F p=\frac{6000 W h / d i ́ a}{150 W}+\frac{6000 W h / d i ́ a}{150 W} * 0.25=\mathbf{5 0}
\end{gathered}
$$

Ecuación 4: Número de paneles requeridos

$$
\begin{aligned}
& \text { Número de panales }=\frac{F p}{\text { hrs sol } / \text { coef } . \text { zona }} \\
& N^{0} \text { paneles }=\frac{50}{8 / 1.15}=7.19 \cong \mathbf{8} \text { paneles }
\end{aligned}
$$

Generación de energía del módulo FV: se analizó mediante la ecuación 2, tomando en cuenta las siguientes consideraciones:

- $\mathrm{P}=150 \mathrm{~W} * 8=1200 \mathrm{~W}$

$\cdot \mathrm{t}=8 \mathrm{~h} / \mathrm{día}$

$\mathrm{E}=\mathrm{P} * \mathrm{t}$ 
$\mathrm{E}=1200 \mathrm{~W} * 8 \mathrm{~h} /$ día $=9600 \mathrm{~W}$ h $/$ día

Se conoce que la potencia necesaria para un trabajo de 8 horas al día es de $6000 \mathrm{Wh} / d i ́ a$. Para obtener dicha cantidad de energía se debe instalar 8 paneles solares de $150 \mathrm{~W}$ c/u conectados en serie, con los datos calculados anteriormente, los parámetros del generador son: potencia total de $1200 \mathrm{~W}$, corriente pico del sistema $8 \mathrm{~A}$, voltaje $165 \mathrm{~V}$, de forma que en 8 horas de trabajo al día se obtendrá una potencia de $9600 \mathrm{~W}$ h/día.

\section{Diseño de estructuras para soporte de paneles solares}

Los soportes están diseñados con tubos rectangulares de dos pulgadas de acero inoxidables, porque es un material que ofrece una tasa de corrosión es de 2.66 micras al año, es decir que la vida útil de la estructura será de 26 años aproximadamente. Además, se tomó en cuanta la orientación e inclinación que de tener.

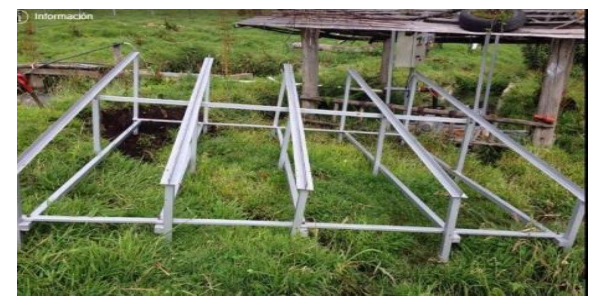

Figura 8. Sistema de mantenimiento

El ángulo de inclinación es de 150 al sureste, como indica la figura 9, de tal forma que sirva para captar la máxima cantidad de radiación solar.

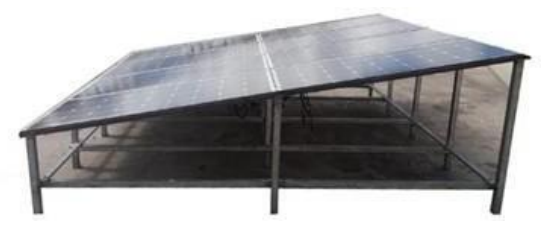

Figura 9. Inclinación e orientación del módulo FV

\section{Montaje de los paneles solares}

Los panales solares están empotrados sobre la parte mecánica de la estructura armada ya fijada con tornillos de acero inoxidable, en cada mesa están ubicados dos paneles, colocados en fila de cuatro, de esta manera se obtiene un montaje total de ocho paneles en todo el sistema de 
generación FV. La figura 10 representa el montaje de los paneles solares en la Finca "El Porvenir".

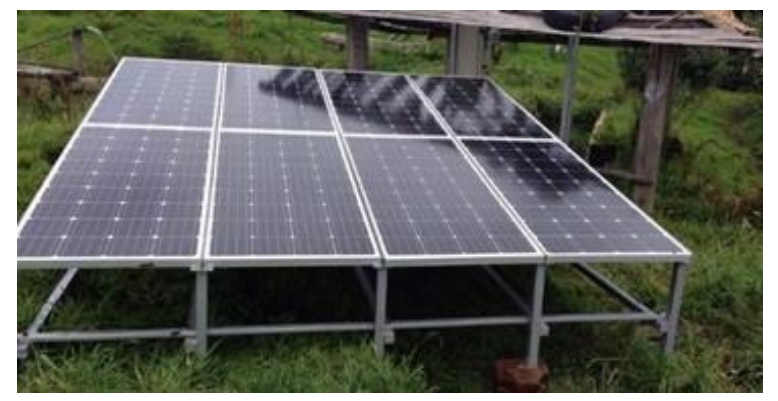

Figura 10. Montaje de los panales

\section{Dimensionamiento del inversor}

Para dimensionar el inversor a utilizar, se considera la corriente y la tensión nominal del motor, garantizando el máximo rendimiento de la instalación fotovoltaica, se debe considerar uno o más hileras de paneles solares conectados en serie, que deberán proporcionar:

- La potencia eléctrica del motor (P1), La potencia fotovoltaica (Wp) debe ser al menos igual a la potencia del motor eléctrico (P1).

- Tensión nominal del motor a la máxima potencia, la tensión nominal de cada hilera fotovoltaica (Vmp) debe ser al menos igual a la tensión nominal del motor multiplicado por el factor de 1,4.

- La tensión de circuito abierto de cada hilera (Voc) debe ser inferior a la tensión de servicio máxima del VASCO Solar.

Cálculos de dimensionamiento del inversor: se debe tomar en cuenta los siguientes datos:

- $\mathrm{P}=746 \mathrm{~W}$

- $\mathrm{I}=3.15 \mathrm{~A}$

$\cdot \mathrm{V}=3 \times 220 \mathrm{~V}$

Siendo la tensión nominal del motor 220 VAC y la corriente nominal de 3.15 A, tomado en consideración estos parámetros y basado en la tabla 1 el modelo más adecuado para aplicación es el inversor VASCO Solar 212. 
Tabla 1. Tipos de inversores

\begin{tabular}{|c|c|c|c|c|c|c|c|}
\hline \multirow{2}{*}{ Modelo } & Vin & $\begin{array}{c}\text { Vin } \\
\text { P1 nom * }\end{array}$ & Max. V out & Max. I out & \multicolumn{2}{|c|}{ P2 Motor * } & Peso \\
\cline { 2 - 9 } & [NDC] & [VDC] & [NAC] & {$[$ A] } & [NAC] & {$[\mathrm{kW}]$} & {$[\mathrm{kg}]$} \\
\hline VASCO Solar 212 & $120-650$ & $>320$ & $3 \times 230$ & 12 & $3 \times 230$ & 2,2 & 8,2 \\
\hline VASCO Solar 409 & $320-850$ & $>560$ & $3 \times 400$ & 9 & $3 \times 400$ & 3 & 8,3 \\
\hline VASCO Solar 412 & $320-850$ & $>560$ & $3 \times 400$ & 12 & $3 \times 400$ & 4 & 8,5 \\
\hline VASCO Solar 415 & $320-850$ & $>560$ & $3 \times 400$ & 15 & $3 \times 400$ & 5,5 & 8,5 \\
\hline VASCO Solar 418 & $320-850$ & $>560$ & $3 \times 400$ & 18 & $3 \times 400$ & 7,5 & 8,5 \\
\hline VASCO Solar 425 & $320-850$ & $>560$ & $3 \times 400$ & 25 & $3 \times 400$ & 11 & 8,5 \\
\hline VASCO Solar 430 & $320-850$ & $>560$ & $3 \times 400$ & 30 & $3 \times 400$ & 15 & 8,7 \\
\hline
\end{tabular}

En la figura 11 se muestra la instalación del inversor VASCO Solar.

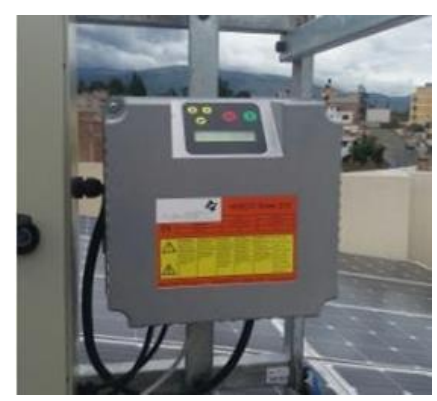

Figura 11. Inversor VASCO solar 212

\section{Programación del Arduino PLC}

La programación del Arduino PLC se realizó en el software Arduino Open-source como se muestra en la figura 12, su funcionamiento es comandar el sistema de bombeo dependiendo de las mediciones realizadas por el sensor, donde internamente en la programación verifica si el valor del oxígeno es menor o igual a 6.4 ppm, mientras esto se cumpla activa el pin 13 la bomba empieza a bombear.

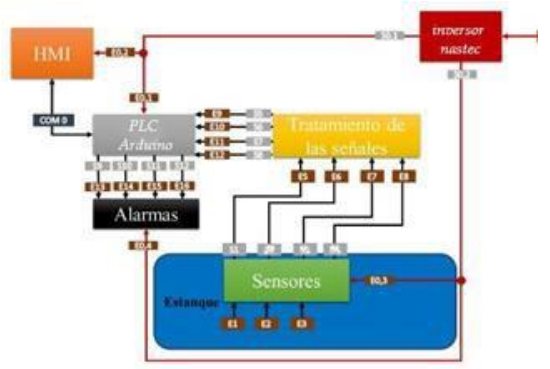

Figura 12. Diagrama de programación 


\section{Implementación de los dispositivos de control y mando}

En esta parte se implementaron todos los dispositivos de mando y control que comandaron el sistema de bombeo, así como también las debidas protecciones con las que cuenta contar el sistema para evitar sobrecargas y cortocircuitos. Como se muestra en la figura 13.

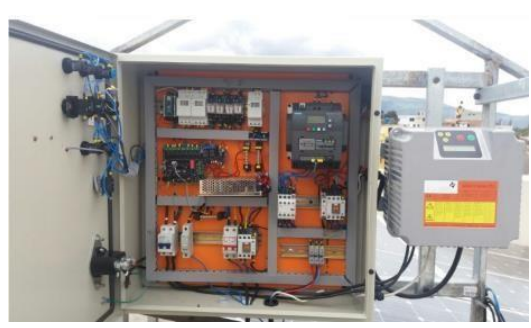

Figura 13. Tablero de control

\section{Instalación del Venturi para la obtención y transferencia de oxígeno en el estanque}

La figura 14 muestra el Tubo Venturi implementado en el sistema para la oxigenación de estanque de truchas. El efecto Venturi consiste en que la corriente de un fluido dentro de un conductor cerrado disminuye la presión del fluido al aumentar la velocidad cuando pasa por una zona de sección menor. Si en este punto del conducto se introduce el extremo del otro conducto se produce una aspiración de flujo contenido en el segundo conducto.

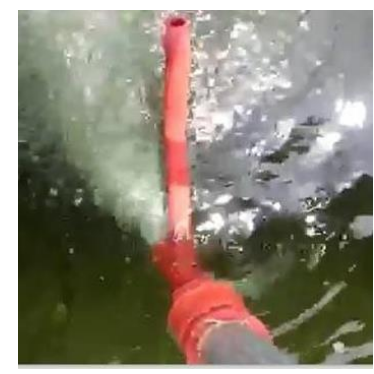

Figura 14. Implementación del Venturi

\section{Análisis de resultados}

Se presentan los resultados obtenidos mediante las mediciones realizadas en el estanque antes y después de la implementación del sistema. Los valores observados representan los promedios de los seis muestreos realizados en cada uno de los cinco días.

El grafico 1 muestra la variación de oxígeno disuelto en el agua del estanque según las muestras tomadas en los 5 días antes de la implantación. 


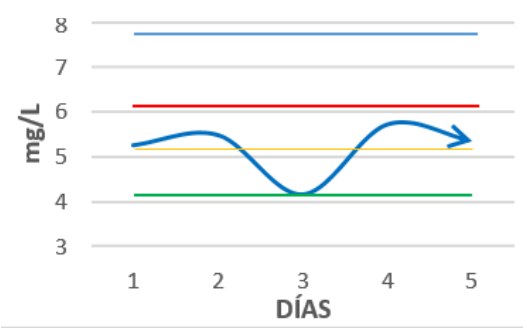

Gráfico 1. Variación del OD antes de la implantación

Según los resultados obtenidos, podemos decir que la concentración del oxígeno se encuentra por debajo del valor mínimo que es de $6.0 \mathrm{mg} / \mathrm{L}$, afectando de esta manera el crecimiento y el desarrollo normal de las truchas.

El grafico 2 muestra la variación de oxígeno disuelto en el agua del estanque según las muestras tomadas en los 5 días después de la implementación.

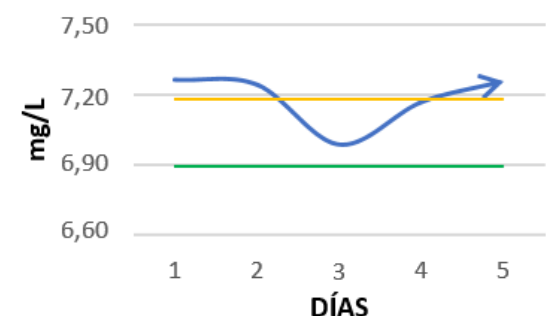

Gráfico 2. Variación del OD después de la implantación

Conociendo que la cantidad de oxígeno disuelto no debe ser menor de $6.0 \mathrm{mg} / \mathrm{L}$, y basándose en los resultados obtenidos, no existen pérdidas significativas de oxígeno en el estanque, teniendo como un máximo y un mínimo de $7.40 \mathrm{mg} / \mathrm{L}$ y $6.93 \mathrm{mg} / \mathrm{L}$ respectivamente lo que se garantiza la producción de oxígeno óptimo para la crianza de truchas.

\section{Análisis de resultados del Oxígeno disuelto en el agua del estanque}

Para el análisis de los resultados se realizó una prueba de hipótesis para medir el incremento de oxígeno disuelto en el estanque de truchas como se detalla a continuación:

Establecer Hipótesis: Planteamos la hipótesis nula y la alternativa:

H_0: La media de las medidas del oxígeno en $\mathrm{mg} / \mathrm{L}$ después de implementar el proyecto es mayor a la media de las medidas del oxígeno en $\mathrm{mg} / \mathrm{L}$ iniciales. 
H_a: La media de las medidas del oxígeno en $\mathrm{mg} / \mathrm{L}$ después de implementar el proyecto es igual o menor a la media de las medidas del oxígeno en $\mathrm{mg} / \mathrm{L}$ iniciales.

De manera simbólica como se muestra en la siguiente ecuación:

Ecuación 1-3: Establecimiento Hipótesis incremento del oxígeno disuelto en el agua después de implementar el proyecto.

H_0: u_T1 $>$ u_T2 y H_a: u_T1 $=<$ u_T2

Dónde:

u_T1: es la media de las medidas del oxígeno en $\mathrm{mg} / \mathrm{L}$ después de implementar el proyecto;

u_T2: es la media de las medidas del oxígeno en $\mathrm{mg} / \mathrm{L}$ antes de implementar el proyecto.

Definición del nivel de significancia: Se tomó un nivel de significancia del 5\%, es decir, $\propto=0,05$.

Número de pruebas y cálculos estadísticos: El número de pruebas realizadas fue de 30, se trata de una prueba bilateral de la media.

Se utilizó el estadístico t de Student para la prueba de hipótesis como se muestra en la tabla 9-3 de los datos previamente al inicio y al final del proyecto.

Tabla 2: Resultados prueba de hipótesis para medir el incremento de oxígeno disuelto en el estanque de truchas.

\begin{tabular}{|l|l|l|}
\hline \multicolumn{3}{|c|}{ Prueba t para medias de dos muestras emparejadas } \\
\hline & OD Final & OD Inicial \\
\hline Media & 7,18533333 & 5,20133333 \\
\hline Varianza & 0,2905154 & 1,04796368 \\
\hline Observaciones & 30 & 30 \\
\hline $\begin{array}{l}\text { Coeficiente de } \\
\text { correlación de Pearson }\end{array}$ & 0,52174376 & \\
\hline $\begin{array}{l}\text { Diferencia hipotética de } \\
\text { las medias }\end{array}$ & 0 & \\
\hline Grados de libertad & 29 & \\
\hline
\end{tabular}




\begin{tabular}{|l|l|l|}
\hline Estadístico t & 12,4428911 & \\
\hline $\mathbf{P}(\mathbf{T}<=t)$ una cola & $\mathbf{1 , 8 6 8 7 E - 1 3}$ & \\
\hline $\begin{array}{l}\text { Valor crítico de t (una } \\
\text { cola) }\end{array}$ & 1,69912703 & \\
\hline $\mathrm{P}(\mathrm{T}<=\mathrm{t})$ dos colas & $3,7375 \mathrm{E}-13$ & \\
\hline $\begin{array}{l}\text { Valor crítico de } \mathrm{t}(\mathrm{dos} \\
\text { colas) }\end{array}$ & 2,04522964 & \\
\hline
\end{tabular}

Decisión estadística: La probabilidad a una cola es mayor que el nivel de significancia, por lo que no se rechaza la $\mathrm{H} \_0$, es decir que la media de las medidas del oxígeno en mg/L después de implementar el proyecto es mayor a la media de las medidas del oxígeno en mg/Liniciales.

De la prueba de hipótesis mediante las medias encontradas, se infiere que existe un incremento de oxígeno disuelto en el agua, los resultados obtenidos son altamente significativos debido a que el valor a una cola es sumamente pequeño lo cual se dice que el sistema implementado tiene un 95\% de confiabilidad al momento de mantener el oxígeno disuelto en el agua dentro de los rangos permitidos para una buena crianza de las truchas.

\section{Conclusiones}

Con la implementación del sistema se tiene un $95 \%$ de confiabilidad al momento de mantener tanto el oxígeno disuelto en el agua como el $\mathrm{pH}$ dentro de los rangos permitidos para una buena crianza de las truchas.

Con el uso de la energía fotovoltaica como una opción para generar fuente de energía se logró eliminar el $\mathrm{CO} 2$ en un $100 \%$ de forma que se contribuye a la no contaminación e impacto ambiental.

Para sistemas de bombeo es recomendable usar motores trifásicos por el consumo de energía menor en un $60 \%$ con respecto a los motores monofásicos. Con respecto a los beneficios que presenta el proyecto es que hay la posibilidad de garantizar al piscicultor un cultivo del $97 \%$, habiendo una pérdida del $3 \%$ con respecto al $20 \%$ de las pérdidas obtenidas con el sistema de bombeo tradicional. 
El proyecto implementado puede ser considerado como una iniciativa para los demás piscicultores de la zona, debido a que la inversión para este sistema se la puede recuperar en un tiempo no mayor a los dos años.

\section{Referencias bibliográficas}

CONELEC. Atlas Solar del Ecuador con fines de Generación Eléctrica. QUITO: CIE, 2008.

CORDOBA NIETO, ERNESTO. Manufactura y automatización. Ingeniería e Investigación. [Consulta: 2015-12-18]. Disponible en: http://www.scielo.org.co/scielo.php?pid=S012056092006000300014\&script=sci_arttext

Daneri, Pablo A. PLC Automatización y control industrial. Buenos Aires-Argentina: Hispano Americana S.A., 2008.

Energía, Centro de Investigación en Energía Renovable. México: s.n., 2010.

CASTEJÓN OLIVA AGUSTIN, Instalaciones solares fotovoltaicas, España, Edinex S.A, 2010.

FERNÁNDEZ JOSÉ, Guía práctica de la energía solar fotovoltaica y termoeléctrica. Madrid: A. Madrid Vicente, 2008.

MAZA CABELLO, Ana Cristina. Diseño de un sistema experto para el enderezado de chasis en frío [En línea] (Tesis) (Pregrado) Universidad de las Américas Puebla. México. 2009. [Citado el: 20 de Enero de 2016.] http://catarina.udlap.mx/u_dl_a/tales/documentos/l $\mathrm{mt} / \mathrm{maza}$ _c_ac/capitulo4.pdf

VELÁSQUEZ, José Antonio. Tecnologías para la Automatización Industrial. [En línea] (Tesis) (Pregrado) Universidad Ricardo Palma. [Consulta: 2016-01-19]. Disponible en:http://www.urp.edu.pe/labcim/portal/imagenes/a rticulo_URP[1].pdf 